\title{
Peter Hazdra
}

\section{Die UNO-Friedensoperation in Kambodscha}

Vorgeschichte, Konzept, Verlauf und kritische Evaluierung des internationalen

Engagements

Europäische Hochschulschriften, Reihe XXXI Politikwissenschaft, Band 322

Peter Lang Verlag, Frankfurt a.M. u.a., 1997, 481 S., DM 118,--

Die Erstfassung der hier zu besprechenden Untersuchung wurde Anfang 1996 von der Rechtswissenschaftlichen Fakultät der Universität Wien als von Hanspeter Neuhold betreute Dissertation angenommen: Sie stellt eine ungewöhnliche Kombination von zeitgeschichtlicher Darstellung und politikwissenschaftlicher sowie staats- und völkerrechtlicher Analyse mit eingestreuten militärischen und wirtschaftlichen Betrachtungen dar, die man zunächst mit Verwunderung zur Kenntnis nimmt. Anders wäre es aber wohl nicht möglich gewesen, Hintergründe und Zusammenhänge eines von der Weltöffentlichkeit nur höchst undeutlich wahrgenommenen Vorgangs darzustellen und zu erklären.

Wie der Verfasser in der Einleitung schreibt, entschloß er sich zu dieser Untersuchung während seines Einsatzes 1992/93 als österreichischer Blauhelmoffizier in Kambodscha, als er auf seinem abgelegenen Beobachterposten im Radio immer wieder von Erfolgen der UNO-Mission hörte, von denen er vor Ort nichts festellen konnte. Die Verbindung der Folgerung aus eigenem Erleben mit den Ergebnissen späteren Aktenstudiums hat hier ein Werk entstehen lassen, dessen Authentizität schwer zu überbieten sein dürfte.

Zunächst schildert Hazdra in einem historischen Rückblick die fortschreitende Schrumpfung Kambodschas von der regionalen Vormacht des Khmer-Reiches von Angkor im 13. Jahrhundert zum Pufferstaat zwischen Siam/Thailand im Westen und Annam/Vietnam im Osten mit ständigen Gebietsverlusten an beide bis zum französischen Protektorat. Dann die Unabhängigkeit 1947, die Neutralitätspolitik Norodom Sihanouks in den beiden Indochinakriegen. Nachdem dieser 1970 mit US-Unterstützung durch General Lon Nol gestürzt worden war, wurde das Land in den Krieg hineingezogen, das Lon-Nol-Regime brach zusammen, die Roten Khmer unter Pol Pot konnten ihre Schreckensherrschaft errichten, bis sie 1979 durch vietnamesische Truppen vertrieben wurden. Diese setzten in Phnom Penh ein ihnen genehmes Regime ein, begannen aber auch mit der Ansiedlung vietnamesischer Bauern in den kambodschanischen Ostprovinzen. Folgerichtig suchten und fanden die Roten Khmer Unterstützung in Thailand, an dessen Grenze sie ein Herrschaftsgebiet behaupten konnten. Auch zwei nichtkommunistische Widerstandsbewegungen konnten sich in kleineren Enklaven halten. Währenddessen erkannte die Staatengemeinschaft die den größten Teil des Landes kontrollierende Regierung in Phnom Penh nicht an, da sie ihre Existenz einer völkerrechtswidrigen Aggression verdankte: Der VN-Sitz Kambodschas blieb den in den Urwald vertriebenen Roten Khmer erhalten.

In dieser völlig verfahrenen Situation brachten drei eher unwahrscheinliche Friedensengel Arafat, Ceausescu und Kim Il-sung (!) - die Streitparteien an einen Tisch, wo sie schließlich nach vierjährigem Hin und Her das Pariser Friedensabkommen vom 23.10.1991 unter- 
zeichneten, nachdem sich die vietnamesische Armee 1989 zurückgezogen hatte. Parteien waren

- die Regierung in der Hauptstadt unter Hun Sen, die sich bis 1989 "People's Republic of Kampuchea", danach "State of Cambodia" (SOC) nannte und sich dann die Regierungspartei "Cambodian People's Party" (CPP) zulegte;

- die Roten Khmer unter Khieu Samphan unter der Bezeichnung "Party of Democratic Kampuchea" (PDK), die sich dann an den Wahlen nicht beteiligten;

- der royalistische "Front Uni National pour un Cambodge Indépendant, Neutre, Pacifique et Coopératif" (FUNCINPEC) unter Sihanouk, später seinem Sohn Ranariddh und

- die "Republikaner" unter Son Sann als "Khmer Peoples National Liberation Front" (KPNLF), die bei den Wahlen als "Buddhist Liberal Democratic Party" (BLDP) auftraten.

Aufgrund des Abkommens erteilten die VN dann das Mandat für die größte, ehrgeizigste und aufwendigste "Friedenserhaltende Operation", die es je gab: Die "United Nations Transitional Authority in Cambodia" (UNTAC) sollte die Regierungsgewalt im Lande übernehmen, die öffentliche Sicherheit garantieren, Wahlen zu einer Verfassungsgebenden Versammlung durchführen, deren Arbeit beratend begleiten und schließlich der aufgrund der neuen Verfassung zu bildenden Regierung die Macht übergeben. Nur zweimal war bis dahin auch nur annähernd Vergleichbares unternommen worden: Mit der schon fast vergessenen UNTEA in West Irian 1962/63 und mit der 1978 eingesetzen, aber erst 1989/90 zum Zuge gekommenen UNTAG in Namibia.

Nachdem das seit 01.11.1991 tätige Vorkommando UNAMIC die nötigen Vorarbeiten geleistet hatte, nahm UNTAC am 15.03.1992 mit dem Eintreffen des japanischen Diplomaten Akashi als Sonderbeauftragter des Generalsekretärs und des australischen Generals Sanderson als Oberbefehlshaber offiziell die Arbeit auf. Bis alle Mitarbeiter (aus 46 Staaten) und das notwendige Material vor Ort waren, vergingen allerdings noch Wochen und Monate. Schließlich umfaßte UNTAC (ohne Ortskräfte) über 21.000 Personen (Namibia knapp 9.000), darunter 15.900 Soldaten aus 34 und 3.600 unbewaffnete Polizisten aus 32 Staaten. Hieraus allein entstanden erhebliche Koordinationsschwierigkeiten, die noch dadurch verschärft wurden, daß die Leiter der einzelnen UNTAC-Komponenten einander erst im Flugzeug von Bangkok nach Phnom Penh kennenlernten. Als alleiniger kambodschanischer Ansprech- und Verhandlungspartner stand UNTAC der nach der Pariser Konferenz gebildete "Supreme National Council" (SNC) unter Vorsitz von Sihanouk zur Verfügung, in dem die vier Konfliktparteien vertreten waren.

In der Folgezeit gelang es UNTAC nicht, neben der für die Verhandlungen gebotenen Flexibilität auch die für die Herstellung und Bewahrung der öffentlichen Ordnung (z.B. landesweite Bewegungsfreiheit) erforderliche Härte aufzubringen, so daß die gesetzten Ziele nur teilweise erreicht werden konnten. So gelang die Kantonierung und Entwaffnung der Bürgerkriegsarmeen nur unvollkommen, vor allem, weil die PDK unter Hinweis auf angeblich im Lande verbliebene Teile der vietnamesischen Armee die Waffenabgabe ablehnte und keine UNTAC-Mitarbeiter in ihr Herrschaftsgebiet ließ. Die Ansiedlung der 
zurückströmenden Flüchtlinge erwies sich als problematisch, weil die vorgesehenen Gebiete zum Teil vermint waren und zum Teil - in der Trockenzeit ausgewählt - nun unter Wasser standen. Wegen des äußerst restriktiv ausgelegten Selbstverteidigungsrechts wurden die Blauhelme - mit Ausnahme des sich nicht an diese Vorgabe haltenden französischen Bataillons - von der Bevölkerung bald als Papiertiger angesehen, und der Zivilverwaltungskomponente gelang es nie, die weitgehend von der CPP dominierte Verwaltung im Text durchgängig als EAS für "Existing Administrative Structure" bezeichnet - wirklich unter Kontrolle zu bringen. Erfolgreich war dagegen die Registrierung von 4,7 Mio. Wahlberechtigten und von 20 Parteien.

Unter diesen Umständen entschloß sich die UNTAC-Spitze trotz Nichterfüllung mehrerer Mandatskriterien und immer wieder aufflammender Kampfhandlungen, die Wahlen durchzuführen, obwohl die sie ohnehin boykottierende PDK mehrfach drohte, sie durch massive Störaktionen zu verhindern. Vom 23. bis 28.05.1993 wurde dann gewählt, wobei sich $89,56 \%$ der registrierten Wähler beteiligten. Die angedrohten Störaktionen der PDK blieben aus, und nicht nur die angereisten internationalen Wahlbeobachter, auch die beteiligten Parteien (nach einer Schamfrist sogar die boykottierende PDK) akzeptierten die Wahl als frei und fair.

Dies änderte sich nach der Stimmauszählung: Nicht die auch wegen ihrer günstigen Placierung als Nr. 1 auf dem Wahlzettel allgemein favorisierte CPP hatte nämlich gewonnen, sondern der eher unauffällig in der Mitte der zweiten Spalte des dreispaltigen Wahlzettels (ein Blick auf dieses als Annex XXI abgedruckte Dokument lohnt sich!) erscheinende FUNCINPEC mit 45,47\% und 58 Mandaten (CPP 38,23\%, 51 Mandate; BLDP 3,81\%, 10 Mandate). Von den Splitterparteien erreichte nur die MOLINAKA mit 1,37\% ein Mandat, die übrigen gingen leer aus. Die folgenden Proteste und Putschdrohungen konnte Sihanouk als SNC-Vorsitzender durch Einsetzung einer im VN-Mandat nicht vorgesehenen Allparteien-Übergangsregierung mit Ranariddh (FUNCINPEC) und Hun Sen (CPP) als gleichberechtigten Premierministern auffangen, was UNTAC und die VN schließlich als "angemessen" akzeptierten.

Trotz zahlreicher Querelen und sich rapide verschlechternder Sicherheitslage verabschiedete die Verfassunggebende Versammlung am 21.09.1993 eine Verfassung, die Kambodscha (amtlich: "Cambodia") als sogar mit einem Verfassungsgerichtshof versehene liberale Mehrparteiendemokratie mit monarchischer Spitze organisiert. Dabei wird der König vom Kronrat (Präsident und 2 Vizepräsidenten der Nationalversammlung, Premierminister, Oberhäupter der 2 buddhistischen Orden) aus den über 30 Jahre alten Mitgliedern der königlichen Familie gewählt. Menschen- und Bürgerrechte werden garantiert, Mehrparteiensystem und Monarchie können auch mit verfassungändernder Mehrheit nicht abgeschafft werden.

Am 24.09. wurde Sihanouk zum König gewählt (seine Frau Monique zur Königin) und setzte Ranariddh als Ersten, Hun Sen als Zweiten Premierminister einer zwischen FUNCINPEC und CPP hälftig aufgeteilten Regierung aus 22 Ministern ein. Mit allgemei- 
nen Glückwünschen versehen, reisten Akashi und Sanderson am 26.09. ab, UNTAC wurde 03.01.1994 abgewickelt. Operation gelungen?

Der Verfasser stellt eine düstere Prognose: Die Realverfassung wich schon bald negativ von der Papierform ab: Die Gewaltenteilung funktioniert nicht, vor allem, weil die CPP die Verwaltung auch dort weiter beherrscht, wo FUNCINPEC-Vertreter als Leiter eingesetzt wurden. Von Pressefreiheit und unabhängiger Justiz kann keine Rede sein, ein Ende der Kampfhandlungen ist nicht abzusehen, wenn auch die PDK nach zahlreichen Desertionen kaum eine Überlebenschance hat.

Die Entmachtung und Exilierung Ranariddhs durch seinen "Kollegen" Hun Sen im Sommer 1997 hat Hazdras pessimistische Erwartungen noch übertroffen. Bedrückend ist auch, daß für den bisher als Integrationsfigur unentbehrlichen krebskranken König Sihanouk noch kein Nachfolger in Sicht ist - außer vielleicht dem als Ballettlehrer und UNESCO-Botschafter in Paris lebenden Prinzen Sihamoni als Kompromißkandidat.

Äußerst kritisch fällt auch im Gegensatz zu dem zunächst überschwenglichen Medienlob die Bewertung des UNTAC-Einsatzes aus. Wegen übertriebener Konfliktscheu der Führung und der generellen Abgehobenheit von der kambodschanischen Realität (Pendeln im klimatisierten Wagen zwischen klimatisiertem Hotel und klimatisiertem Büro) sei manche Möglichkeit helfenden Eingreifens vertan worden. Hinzu kamen interne Spannungen zwischen dem diplomatisch geschulten, aber fachunkundigen VN-Personal und den von den Staaten entsandten Experten, sowie die extreme Ungleichheit in der Ausstattung und Bezahlung der militärischen Kontingente (das japanische Bataillon z.B. glaubte, ohne Badehäuser mit elektronisch gesteuerter Wassertemperatur nicht auskommen zu können). Die Zivilpolizei schließlich sei, da unbewaffnet, völlig nutzlos und dazu grotesk überbezahlt (\$130,- Tagegeld) gewesen. Auch der mit dem UNTAC-Einsatz zunächst einhergehende Wirtschaftsaufschwung habe sich nach Abzug des VN-Personals weitgehend als eine vergängliche Sumpfblüte aus Bauboom, Korruption, Prostitution und Kriminalität erwiesen, verstärkt durch das Fehlverhalten mancher UNTAC-Angehöriger, das fast nie geahndet worden sei.

Abschließend macht der Verfasser noch Vorschläge für die Planung und Personalausstattung künftiger VN-Einsätze, von denen er allerdings selbst annimmt, daß sie wegen der divergierenden Interessen der Mitgliedstaaten wenig Chancen auf Beachtung haben.

Insgesamt entsteht der Eindruck, daß sich der Verfasser mit seinem Vorhaben etwas übernommen hat. Trotzdem wird niemand an dieser Studie vorbeikommen, der oder die sich für neuere Geschichte und Recht Kambodschas interessiert oder die höchst aktuelle Problematik von VN-Friedenseinsätzen allgemein oder im konkreten Einzelfall erforschen will.

Karl Leuteritz 